OPEN ACCESS

Edited by:

Zhenyu Li,

Deakin University, Australia

Reviewed by:

Fuping Zeng,

Wuhan University, China

Peng Li,

King Abdullah University of Science and Technology, Saudi Arabia

*Correspondence:

Qu Zhou

zhouqu@swu.edu.cn

Specialty section: This article was submitted to

Nanoscience,

a section of the journal

Frontiers in Chemistry

Received: 19 April 2018

Accepted: 14 May 2018

Published: 30 May 2018

Citation:

Zhou Q, Lu Z, Wei Z, Xu L, Gui Y and

Chen W (2018) Hydrothermal

Synthesis of Hierarchical Ultrathin $\mathrm{NiO}$ Nanoflakes for High-Performance $\mathrm{CH}_{4}$

Sensing. Front. Chem. 6:194

doi: 10.3389/fchem.2018.00194

\section{Hydrothermal Synthesis of Hierarchical Ultrathin NiO Nanoflakes for High-Performance $\mathrm{CH}_{\mathbf{4}}$ Sensing}

\author{
Qu Zhou ${ }^{1,2 *}$, Zhaorui Lu ${ }^{1}$, Zhijie Wei ${ }^{1}$, Lingna Xu ${ }^{1}$, Yingang Gui ${ }^{1}$ and Weigen Chen ${ }^{2}$ \\ ${ }^{1}$ College of Engineering and Technology, Southwest University, Chongqing, China, ${ }^{2}$ State Key Laboratory of Power \\ Transmission Equipment \& System Security and New Technology, Chongqing University, Chongqing, China
}

Keywords: hydrothermal synthesis, ultrathin NiO nanoflakes, methane, gas sensor, sensing performances

\section{INTRODUCTION}

Methane $\left(\mathrm{CH}_{4}\right)$, as a colorless and odorless gas, is the main component of natural gas and widely used in various industries and human daily life (Schoonbaert et al., 2015). However, the leakage of natural gas, oil and gas storage, transportation and distribution systems increase atmospheric $\mathrm{CH}_{4}$ concentration levels and lead to serious climate changes, which must be addressed (Zheng et al., 2017). Additionally, $\mathrm{CH}_{4}$ will be easy to explode in a range of concentration (5-15\%). Therefore, it is necessary to develop rapid and accurate gas sensors for $\mathrm{CH}_{4}$ detection.

Nickel oxide $\mathrm{NiO}$ (Sun et al., 2014) is a significant p-type semiconductor, and has been widely used as catalyst (Yu et al., 2015), lithium-ion battery (Gu et al., 2016; Long et al., 2018), gas sensor (Wang et al., 2016; Zhou et al., 2018b), magnetic material (Cui et al., 2011), and so on. In recent years, many researchers have reported that $\mathrm{NiO}$ can be applied to fabricate high performance gas sensors for detecting some special gases such as hydrogen (Sta et al., 2016), $\mathrm{NO}_{2}$ (Hoa and El-Safty, 2011), ethanol (Miao et al., 2017), etc. Zhang et al. studied a methane gas sensor based on nickel oxide $(\mathrm{NiO}) /$ reduced graphene oxide $(\mathrm{rGO})$ nanocomposite film, which exhibited a response of $15 \%$ toward $1000 \mathrm{ppm} \mathrm{CH}_{4}$ gas at $260^{\circ} \mathrm{C}$ (Zhang et al., 2016), and the sensing response of the pure $\mathrm{NiO}$ film sensor only was $2.5 \%$ under the same condition. Moreover, few reports about the synthesis of hierarchical $\mathrm{NiO}$ nanostructures and its application for $\mathrm{CH}_{4}$ detection was reported recently.

Thus, in this study we reported the successful synthesis of hierarchical ultrathin $\mathrm{NiO}$ nanoflakes and systematically researched their gas sensing properties to $\mathrm{CH}_{4}$. Interestingly, the proposed sensor exhibited high sensitivity, low optimal operating temperature, good linear relationship and excellent selectivity to $\mathrm{CH}_{4}$.

\section{EXPERIMENTAL DESIGN, MATERIALS, AND METHODS}

\section{Sample Synthesis}

All raw chemicals used for the synthesis of hierarchical ultrathin $\mathrm{NiO}$ nanoflakes were analytical graded and used as received without further purifications. In a typical hydrothermal procedure, $3 \mathrm{mmol}$ of nickel nitrate hexahydrate $\mathrm{Ni}\left(\mathrm{NO}_{3}\right)_{2} \bullet 6 \mathrm{H}_{2} \mathrm{O}$ and $0.200 \mathrm{~g}$ of polyvinylpyrrolidone (PVP) were dissolved in $100 \mathrm{~mL}$ DI deionized water under continuous stirring. After $15 \mathrm{~min}$ 
of rigorous stirring, a few drops of $\mathrm{NH} 4 \mathrm{OH}$ solution were added to the resultant solution to maintain the $\mathrm{pH}=11$. The mixture was stirred vigorously for $30 \mathrm{~min}$ and then transferred into a Teflon-lined stainless steel autoclave, sealed and heated to $180^{\circ} \mathrm{C}$ for $6 \mathrm{~h}$. After cooling to room temperature naturally, the product was collected by centrifugation and washed with DI water and ethanol several times, respectively, and dried at $60^{\circ} \mathrm{C}$ overnight. Finally, the dried power was calcined at $400^{\circ} \mathrm{C}$ for $2 \mathrm{~h}$.

\section{Sample Characterization}

The phase structure of the prepared products were characterized by X-ray diffraction (XRD) using a Rigaku D/Max-1200X diffractometry with $\mathrm{Cu}-\mathrm{K} \alpha$ radiation operated at $30 \mathrm{KV}$ and $100 \mathrm{~mA}$. The morphologies, microstructures and elemental compositions of the synthesized samples were investigated with a Nova 400 Nano field emission scanning electronic microscopy (FE-SEM), equipped with an energy dispersive X-ray spectroscopy (EDS). Gas sensors were fabricated with the side heated structure (Zhou et al., 2018c) and gas sensing properties of the obtained sensors were performed with the CGS-8 (Chemical gas sensor-8) intelligent gas sensing analysis system (Beijing Elite Tech Co., Ltd., Beijing, China).

\section{RESULTS}

\section{Materials Characterization}

Figure 1A shows the XRD pattern of the synthesized $\mathrm{NiO}$ sample. As shown, all the primary diffraction peaks observed at $37.30^{\circ}$, $43.25^{\circ}, 62.85^{\circ}, 75.45^{\circ}$, and $79.20^{\circ}$ could be well assigned to (111), (200), (220), (311), and (222) planes of the cubic form of NiO (JCPDS Card No. 47-1049). Figure 1B depicts the EDS spectrum of the prepared $\mathrm{NiO}$ sample. As demonstrated, only nickel $(\mathrm{Ni})$ and oxygen $(\mathrm{O})$ peaks are observed with $\mathrm{O} / \mathrm{Ni}$ molar ratio of nearly 1:1. No other diffraction peaks from impurities and dispersive peaks related with any element were observed, indicating a high purity of the as-prepared hierarchical ultrathin $\mathrm{NiO}$ nanoflakes sample.

Figures 1C,D demonstrate the FESEM image of the synthesized hierarchical $\mathrm{NiO}$ nanostructures, which are constructed by many ultrathin nanoflakes with smooth surface. The diameter of the $\mathrm{NiO}$ nanoflakes is in the scope of 300 to $400 \mathrm{~nm}$ with thickness ranging from 10 to $15 \mathrm{~nm}$.

\section{Sensing Performances}

The gas response of the fabricated side-heated sensor is defined as $\mathrm{Rg} / \mathrm{Ra}$, where $\mathrm{Ra}$ and $\mathrm{Rg}$ are the resistance values of the sensor in air and in the tested gas, respectively (Zeng et al., 2012; Zhou et al., 2018b). Figure 2A shows the relationship between the operating temperature and the gas response of the sensor to $30 \mathrm{ppm}$ of $\mathrm{CH}_{4}$ with working temperature ranging from 100 to $350^{\circ} \mathrm{C}$. As can be seen, with the increase of the temperature, the sensing response increases at first and attains its maximum value, and then decreases rapidly with further increasing temperature. The optimum operating temperature of the sensor to $\mathrm{CH}_{4}$ is measured to be about $225^{\circ} \mathrm{C}$, lower than some already reported results (Zhang et al., 2016), and the corresponding response is 46.53 .

Figure 2B illustrates the gas response of the sensor to various concentration of $\mathrm{CH}_{4}$ ranging from 0.2 to $50 \mathrm{ppm}$ at $225^{\circ} \mathrm{C}$. It is apparent that the sensing response increases rapidly with increasing gas concentration and a good linear relationship
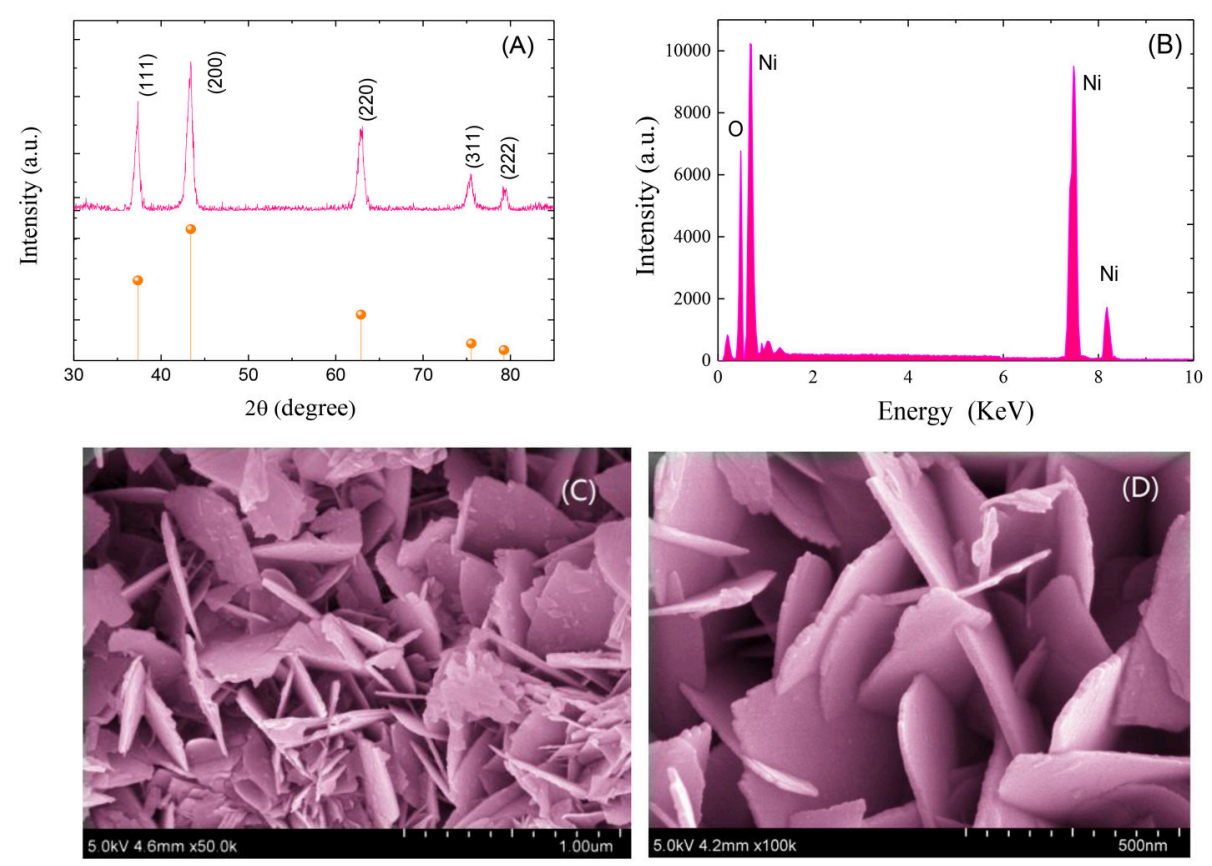

FIGURE 1 | XRD patterns (A), EDS patterns (B) and FESEM images (C,D) of the synthesized hierarchical ultrathin NiO nanoflakes. 

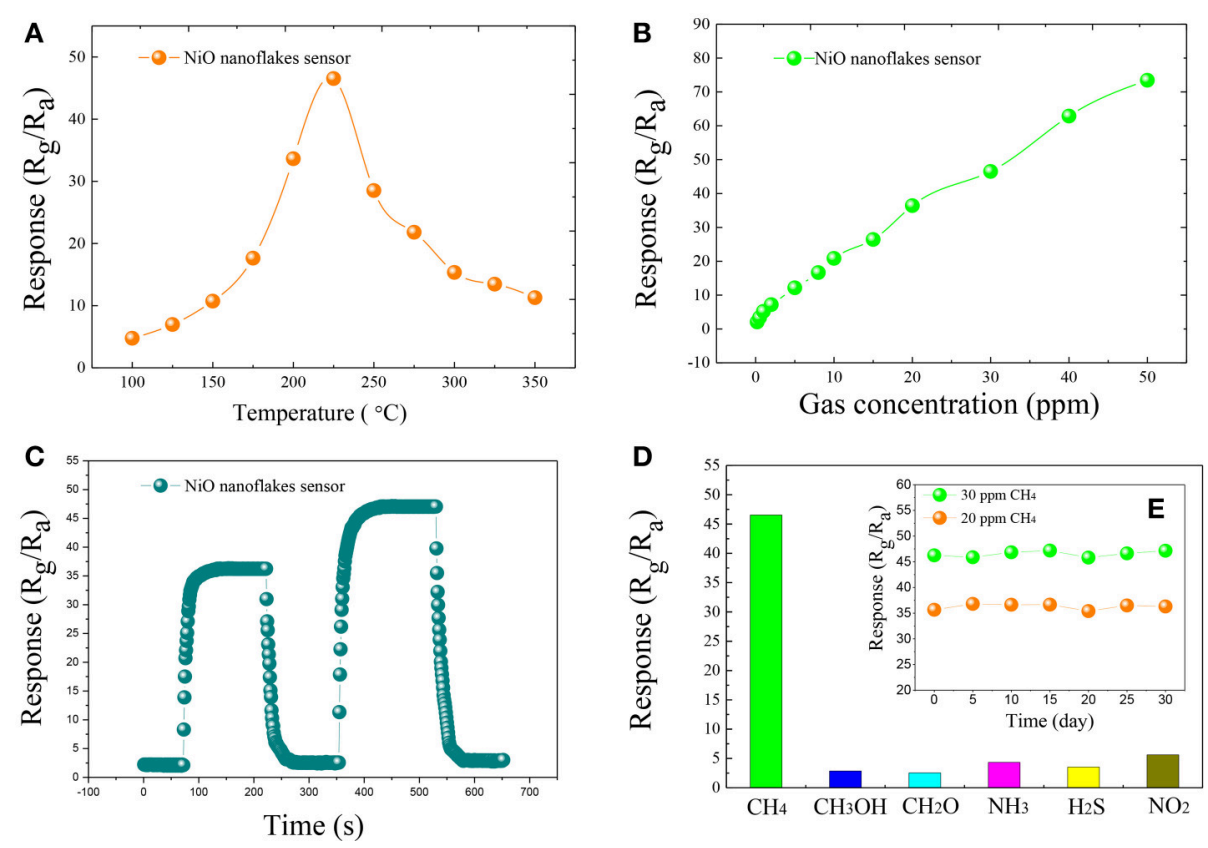

FIGURE 2 | The gas response of the synthesized $\mathrm{NiO}$ nanoflakes sensor toward $30 \mathrm{ppm}$ of $\mathrm{CH}_{4}$ at different working temperatures (100-350 ${ }^{\circ} \mathrm{C}$ ( $\mathbf{A}$ ), gas response of the synthesized ultrathin $\mathrm{NiO}$ nanoflakes sensor to various concentration $\mathrm{CH}_{4}$ in the range from 0.2 to 50 ppm at $225^{\circ} \mathrm{C}(\mathbf{B})$, the response and recovery curves of the synthesized ultrathin $\mathrm{NiO}$ nanoflakes sensor to 20 and 30 ppm $\mathrm{CH}_{4}$ at $225^{\circ} \mathrm{C}(\mathbf{C})$, the response of $\mathrm{NiO}$ nanoflakes sensor toward 30 ppm of different testing gases at $225^{\circ} \mathrm{C}$ (D), and the long-term stability of $\mathrm{NiO}$ nanoflakes sensor to 20 and $30 \mathrm{ppm}^{\mathrm{CH}} \mathrm{H}_{4}$ at $225^{\circ} \mathrm{C}$ (E).

between the sensing response and gas concentration can be obtained, implying an effective candidate for low concentration $\mathrm{CH}_{4}$ detection.

Figure 2C demonstrates the dynamic response and recovery curve of the as-prepared sensor to $20,30 \mathrm{ppm} \mathrm{CH}_{4}$ at $225^{\circ} \mathrm{C}$. As illustrated, the sensor response increases dramatically when $\mathrm{CH}_{4}$ gas was injected into the test chamber, and rapidly turns back to its initial state when subjected to air for sensor recovering. The time taken by the sensor to reach $90 \%$ of the total resistance change was defined as the response (recovery) time in the case of gas adsorption (desorption). According to this definition (Li et al., 2016; Zhang et al., 2018; Zhou et al., 2018a), the response and recovery time of the $\mathrm{NiO}$ sensor toward $30 \mathrm{ppm} \mathrm{CH} 4$ are calculated to be about $15 \mathrm{~s}$ and $20 \mathrm{~s}$, respectively.

Figure 2D depicts the sensing response histogram of the $\mathrm{NiO}$ sensor to $30 \mathrm{ppm}$ of various gases, including $\mathrm{CH}_{3} \mathrm{OH}, \mathrm{CH}_{2} \mathrm{O}, \mathrm{NH}_{3}, \mathrm{H}_{2} \mathrm{~S}$, and $\mathrm{NO}_{2}$. It can be seen that the presented $\mathrm{NiO}$ sensor shows extremely high response to $\mathrm{CH}_{4}$ than other potential interfering gases. The longterm stability of the sensor was also measured and shown in Figure 2E, inserted in Figure 2D, where the sensor response changes slightly and keeps at a nearly constant value during the long experimental cycles, implying an excellent longtime stability and repeatability of the sensor for $\mathrm{CH}_{4}$ detection.

It is known to all that $\mathrm{NiO}$ is a typical p-type semiconducting material, and its sensing properties are predominantly controlled by the surface resistance (Wang et al., 2016). When the fabricated $\mathrm{NiO}$ nanoflakes sensor is exposed to air, oxygen molecules would capture free electrons to form chemisorbed oxygen in the form of $\mathrm{O}_{2 \text { ads }}^{-}, \mathrm{O}_{\text {ads }}^{-}$and $\mathrm{O}_{\text {ads }}^{2-}$ absorbed on the sensor surface, increasing the number of electron holes of $\mathrm{NiO}$ surface and its conductivity. In $\mathrm{CH}_{4}$ gas ambient, oxidation-reduction reactions would take place between the pre-adsorbed oxygen ions and $\mathrm{CH}_{4}$ molecules, and then electrons are released back to $\mathrm{NiO}$ electron holes, resulting in a decreasing conductivity of the $\mathrm{NiO}$ nanoflakes sensor.

\section{CONCLUSIONS}

In summary, hierarchical ultrathin $\mathrm{NiO}$ nanoflakes were successfully synthesized via hydrothermal process and characterized by XRD, FESEM and EDS, for the purpose of fabricating highly sensitive $\mathrm{CH}_{4}$ gas sensors. The diameter of the prepared $\mathrm{NiO}$ nanoflakes material is in the scope of 300 to $400 \mathrm{~nm}$ with thickness ranging from 10 to $15 \mathrm{~nm}$. Side-heated gas sensor was fabricated with the synthesized ultrathin $\mathrm{NiO}$ nanoflakes and methane $\mathrm{CH}_{4}$ sensing performances were systematically evaluated. The synthesized hierarchical ultrathin $\mathrm{NiO}$ nanoflakes sensor exhibited high sensitivity, low optimal operating temperature, rapid response and recovery time, excellent selectivity and stability to $\mathrm{CH}_{4}$ gas. Moreover, good linear relationship between the sensing response and gas 
concentration from 0.2 to $50 \mathrm{ppm}$ was also obtained. All results indicate that the synthesized hierarchical ultrathin $\mathrm{NiO}$ nanoflakes may be a potential sensing material for fabricating high performance gas sensor for low concentration $\mathrm{CH}_{4}$ detection.

\section{AUTHOR CONTRIBUTIONS}

QZ and ZL performed the experiments and analyzed the data with the help from ZW and YG. QZ and ZL wrote the manuscript

\section{REFERENCES}

Cui, Y. F., Wang, C., Wu, S. J. G., Liu, Zhang, F. F., and Wang, T. M. (2011). Lotus-root-like $\mathrm{NiO}$ nanosheets and flower-like $\mathrm{NiO}$ microspheres: synthesis and magnetic properties. CrystEngComm 13, 4930-4934. doi: 10.1039/ clce05389b

Gu, L. L., Xie, W. H., Bai, S. A., Liu, B. L., Xue, S., Li, Q., et al. (2016). Facile fabrication of binder-free $\mathrm{NiO}$ electrodes with high rate capacity for lithium-ion batteries. Appl. Surf. Sci. 368, 298-302. doi: 10.1016/j.apsusc. 2016.01.270

Hoa, N. D., and El-Safty, S. A. (2011). Synthesis of mesoporous NiO nanosheets for the detection of toxic NO2 gas. Chem. Eur. J. 17, 12896-12901. doi: 10.1002/chem.201101122

Li, T. M., Zeng, W., Long, H. W., and Wang, Z. C. (2016). Nanosheet-assembled hierarchical $\mathrm{SnO} 2$ nanostructures for efficient gas-sensing applications. Sens. Actuators B 231, 120-128. doi: 10.1016/j.snb.2016.03.003

Long, H., Zeng, W., Wang, H., Qian, M. M., Liang, Y. H., and Wang, Z. C. (2018). Self-assembled biomolecular 1D nanostructures for aqueous SodiumIon battery. Adv. Sci. 5:1700634. doi: 10.1002/advs.201700634

Miao, R. Y., Zeng, W., and Gao, Q. (2017). Hydrothermal synthesis of novel NiO nanoflowers assisted with CTAB and SDS respectively and their gas-sensing properties. Mater. Lett. 186, 175-177. doi: 10.1016/j.matlet.2016.09.127

Schoonbaert, S. B., Tyner, D. R., and Johnson, M. R. (2015). Remote ambient methane monitoring using fiber-optically coupled optical sensors. Appl. Phys. B 119, 133-142. doi: 10.1007/s00340-014-6001-0

Sta, I., Jlassi, M., Kandyla, M., Hajji, M., Koralli, P., Krout, F., et al. (2016). Surface functionalization of solegel grown $\mathrm{NiO}$ thin films with palladium nanoparticles for hydrogen sensing. Int. J. Hydrogen Energ. 41, 3291-3298. doi: 10.1016/j.ijhydene.2015.12.109

Sun, X., Liu, Y. X., Gao, H. Y., Gao, P. X., and Lei, X. (2014). Bimodular high temperature planar oxygen gas sensor. Front. Chem. 2:57. doi: $10.3389 /$ fchem. 2014.00057

Wang, J., Zeng, W., and Wang, Z. C. (2016). Assembly of 2D nanosheets into 3D flower-like NiO: synthesis and the influence of petal thickness on gas-sensing properties. Ceram. Int. 42, 4567-4573. doi: 10.1016/j.ceramint.2015.11.150

Yu, F., Xu, X. L., Peng, H. G., Yu, H. J., Dai, Y. F., Liu, W. M., et al. (2015). Porous $\mathrm{NiO}$ nano-sheet as an active and stable catalyst for $\mathrm{CH} 4$ deep oxidation. App. Catal. A 507, 109-118. doi: 10.1016/j.apcata.2015.09.023 with input from all authors. All authors read and approved the manuscript.

\section{ACKNOWLEDGMENTS}

This work has been supported in part by the National Natural Science Foundation of China (Nos. 51507144, 5127785), China Postdoctoral Science Foundation funded project (Nos. 2015M580771, 2016T90832) and the Chongqing Science and Technology Commission (CSTC) (No. cstc2016jcyjA0400).

Zeng, W., Liu, T. M., and Wang, Z. C. (2012). Enhanced gas sensing properties by $\mathrm{SnO} 2$ nanosphere functionalized $\mathrm{TiO} 2$ nanobelts. J. Mater. Chem. 22, 3544-3548. doi: 10.1039/c2jm15017d

Zhang, D. Z., Chang, H. Y., Li, P., and Liu, R. (2016). Characterization of nickel oxide decorated-reduced graphene oxide nanocomposite and its sensing properties toward methane gas detection. J. Mater. Sci. Mater. El. 27, 3723-3730. doi: 10.1007/s10854-015-4214-6

Zhang, Y. X., Zeng, W., Ye, H., and Li, Y. Q. (2018). Enhanced carbon monoxide sensing properties of $\mathrm{TiO} 2$ with exposed (001) facet: a combined first-principle and experimental study. Appl. Surf. Sci. 443, 507-516. doi: 10.1016/j.apsusc.2018.02.036

Zheng, C. T., Ye, W. L., Sanchez, N. P., Li, C. G., Dong, L., Wang, Y. D., et al. (2017). Development and field deployment of a mid-infrared methane sensor without pressure control using interband cascade laser absorption spectroscopy. Sens. Actuators B 244, 365-372. doi: 10.1016/j.snb.2016.12.146

Zhou, Q., Chen, W. G., Xu, L. N., Kumr, R., Gui, Y. G., Zhao, Z. Y., et al. (2018a). Highly sensitive carbon monoxide (CO) gas sensors based on $\mathrm{Ni}$ and $\mathrm{Zn}$ doped $\mathrm{SnO} 2$ nanomaterials. Ceram. Int. 44, 4392-4399. doi: 10.1016/j.ceramint.2017.12.038

Zhou, Q., Umar, A., Sodki, E. M., Amine, A., Xu, L. N., Gui, Y. G., et al. (2018b). Fabrication and characterization of highly sensitive and selective sensors based on porous $\mathrm{NiO}$ nanodisks. Sens. Actuators B 259, 604-615. doi: 10.1016/j.snb.2017.12.050

Zhou, Q., Xu, L. N., Umar, A., Chen, W. G., and Kumr, R. (2018c). Pt nanoparticles decorated $\mathrm{SnO} 2$ nanoneedles for efficient $\mathrm{CO}$ gas sensing applications. Sens. Actuators B 256, 656-664. doi: 10.1016/j.snb.2017.09.206

Conflict of Interest Statement: The authors declare that the research was conducted in the absence of any commercial or financial relationships that could be construed as a potential conflict of interest.

Copyright (c) $2018 \mathrm{Zhou}, \mathrm{Lu}, \mathrm{Wei}, \mathrm{Xu}$, Gui and Chen. This is an open-access article distributed under the terms of the Creative Commons Attribution License (CC $B Y)$. The use, distribution or reproduction in other forums is permitted, provided the original author(s) and the copyright owner are credited and that the original publication in this journal is cited, in accordance with accepted academic practice. No use, distribution or reproduction is permitted which does not comply with these terms. 